\title{
Dynamic Pricing Scheme for Effective Bandwidth Utilization in Mobile WiMAX
}

\author{
Kafilu Barau Danbatta \\ Nigerian Communications Commission \\ Plot 426 Aguiyi Ironsi Street Maitama Abuja
}

\author{
Umar Garba Danbatta \\ Department of Electrical Engineering \\ Bayero University Kano
}

\begin{abstract}
In the next Generation Networks like Mobile WiMAX, it is highly essential to create a market mechanism that would allow the customer to communicate with Network and negotiate a contract based on some QoS parameters like blocking probability, delay, arrival rate, spectral efficiency, resource allocation and price. However, the mechanisms, rather than technical-oriented scheme, that involve the use of economic theories may provide better solutions to accommodate the high demand of mobile services. The purpose of this research work is to propose and validate mathematical model that study the effect of pricing incentives as an additional strategy for encouraging a more efficient usage of limited network resources. A modified efficient dynamic pricing scheme for optimal network resource utilization in Mobile WiMAX network has been developed and validated. The percentage improvement of the Cumulative Revenue (CR) generated by the proposed model over the existing model ranges between $25 \%$ and $150 \%$ depending on the values of the Price Leveling Factor (PLF). The percentage improvement of the Cumulative Resource Efficiency Index (CREI) generated by the proposed model over the existing model ranges between $6 \%$ and $7.1 \%$ depending on the values of the Price Leveling Factor (PLF). The proposed scheme proved to generate more revenue per Bandwidth Utilization than the existing model.
\end{abstract}

General Terms: Price Leveling Factor (PLF), Total Revenue Generated, Total Allocated Resources, Cumulative Resource Allocated (CRA). Incentive Price, Incentive Cut-off, Threshold for Bandwidth Utilization

Keywords: Cumulative Resource Efficiency Index (CRI), Cumulative Revenue (CR), Acceptance Probability, Utility, Low Priority User (LPU) and High Priority User (HPU). Bandwidth Utilization (BU)

\section{INTRODUCTION}

WiMAX is wireless technology, based on the IEEE 802.16 standard, for nomadic and mobile data access The IEEE 802.16-2004 standard deals with fixed wireless broadband access, while the IEEE 802.16e standard deals with both fixed and mobile wireless broadband access. WiMAX offers costeffective and quickly deployable alternative to cable and DSL networks. It provides high bandwidth and various levels of quality of services (QOS) for different classes of traffic. [1].

Figure 1 shows the functionality of the proposed model. The three QoS classes is considered in order to have a heterogeneous WiMAX environment with real-time and nonreal-time users: Unsolicited Grant Service (UGS), rtPS, and Best Effort (BE). The UGS and rtPS classes support real-time service flows that have fixed-size and variable size data packets on a periodic basis, respectively. The IEEE 802.16e standard considers five QoS classes in order to have a heterogeneous WiMAX environment with real-time and nonreal-time users: Unsolicited Granted Service (UGS), RealTime Polling Service (rtPS), Extended Real-Time Polling Service (ertPS), Non-Real Time Polling Service (nrtPS), and Best Effort (BE). The UGS supports real-time traffic that periodically generates packets of fixed length, such as Voice over IP without silence suppression. The rtPS supports realtime traffic that periodically generates packets of variable length, such as MPEG video. The ertPS supports real-time traffic that periodically generates packets of variable length, such as Voice over IP services with silence suppression. The nrtPS supports non-real time traffic that generates packets of variable length and tolerant to delay, such as FTP. The BE service supports traffic that does not require throughput or delay guarantees, such as HTTP. (Mowafi M. Y., et al. 2012). The BE class, designed for non-real-time applications, has no QoS guarantees and then should be identified as LPU and they are evidently be the cheapest one [3].The other three QoS classes in this paper shall be identified as HPUs.

In WDCRS, the total bandwidth is divided among the service classes. The choice of division is to be an optimization problem to get the maximum utilization of the bandwidth. The three higher-priority classes (UGS, rtPS, ertPS) can be studied separately, since each class has fixed channel allocation pattern. For two lower-priority classes (nrtPS and $\mathrm{BE}$ ), they are treated as two separate cognitive radio users, and the analysis is based on the fact that nrtPS scans the resources first, followed by the BE class[4].

This paper presents a new bandwidth management scheme to obtain high channel utilization and to increase the total throughput in Mobile WiMAX systems to the lower-priority service classes in order to improve the bandwidth utilization without affecting the QoS of the higher-priority classes.

\section{MODEL ASSUMPTIONS}

In the proposed model, it is assumed that each network element incorporates a load monitor so that BW price can be set based on its current load level.

The WiMAX BS-centric framework is based on the following assumptions:

- Real-time on-demand temporary wireless access for LPUs;

- HPUs and LPUs are harmoniously coexisting on similar power levels at the same BS, where HPU service is not affected by LPU access;

- Generation of additional revenue for the WSPs per BS from such LPU access;

- Minimal enhancements are required for implementation in the current Mobile WiMAX architecture. 
- The HPUs and LPUs should not necessarily have cognitive capabilities.

\section{SYSTEM MODEL}

In this section, a mathematical model of price, utility and partitioned based resource CAC for static and dynamic congestion control is developed. Firstly, the CAC algorithm completely partitioned the users according to the five WiMAX classes and prioritize the traffic into Low Priority Users and Higher Priority Users [3]. The nrtPS and BE are the two lower-priority classes while the UGS, rtPS, and ertPS are the three higher priority classes, each with different price, utility and acceptance probability functions. Secondly, each class is attended independently. The overall revenue, the total allocated resources and resource efficiency indices are developed for final performance analysis. LPUs (Low Priority Users) are charged based on the current HPU (High Priority Users) utilization, like $30 \%, 40 \%, 50 \%$ etc. The higher the HPU utilization, the higher the price quoted to the LPUs. The lower the HPU utilization the lower the price quoted to the LPUs. When the LPU utilization is above the incentive cut-off point, then, the charging scheme imposes a monetary penalty by charging LPUs above incentive HPU reference prices. This induces negative incentive to utilize the network at this point [4].

\subsection{Additional Revenue Generated}

The additional revenue is being depicted by equation (5). This is the additional revenue generated by the LPUs in the incentive region. In the incentive region users are encouraged to utilize more bandwidth as they are charged with a price less than the HPU reference price. In the congestion region, the price is raised and approaches infinity. The $p_{\text {inct }}^{L P U}$ Incentive Price payable by admitted Low Priority User was computed by [4], as:

$$
f(\alpha(t))=-\log \left(1-\left(\frac{\alpha(t)}{\alpha_{i, w}^{i c}}\right)^{\delta}\right)
$$

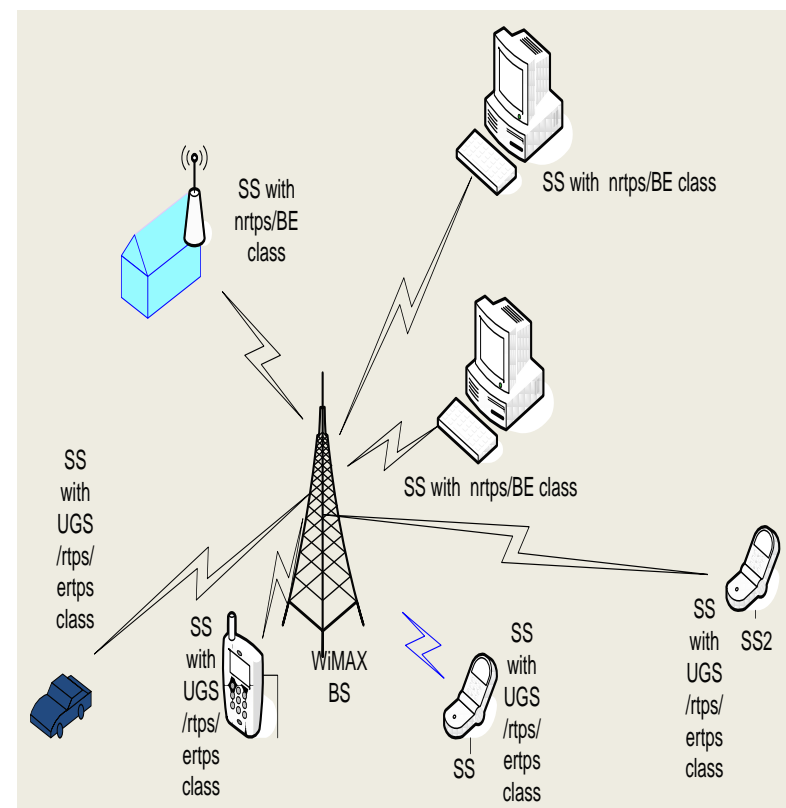

Figure 1: The Schematic Representation of the Proposed Dynamic Congestion Control Model based on Bandwidth Resource Utilization
The pricing for LPUs to gain temporary wireless access to a BS during the small time interval $T f-T s$ (in minutes) was also given by [4], as:

$$
\begin{gathered}
P_{\text {util }}^{L P U}=P_{a c c e s}^{H P U}+\left(P_{\text {inct }}^{H P U}-P_{\text {acces }}^{H P U}\right)[-\log (1 \\
\left.\left.-\left(\frac{\alpha(t)}{\alpha_{i, w}^{i c}}\right)^{\delta}\right)\right]^{m_{i, w}^{i n c t}}
\end{gathered}
$$

It must be noted that the range of LPU prices will remain constant only for a fixed small time interval $T=T f-T s$ (in minutes), where $T s$ indicates the start of the interval and $T f$ the finish [4].

The proposed modified $p_{\text {inct }}^{L P U}$ Incentive Price payable by admitted Low Priority User can be computed as:

$f(\alpha(t))=-\log \left(1-\left(\frac{\alpha(t)-\alpha_{i, w}^{H P U}}{\alpha_{i, w}^{i c}-\alpha_{i, w}^{H P U}}\right)^{\delta}\right)$

The proposed modified pricing for LPUs to gain temporary wireless access to a BS during the small time interval $T f-T s$ (in minutes) can be computed as:

$$
\begin{array}{r}
P_{\text {util }}^{L P U}=P_{\text {acces }}^{L P U}+\left(P_{\text {inct }}^{H P U}-P_{a c c e s}^{L P U}\right)[-\log (1 \\
\left.\left.-\left(\frac{\alpha(t)-\alpha_{i, w}^{H P U}}{\alpha_{i, w}^{i c}-\alpha_{i, w}^{H P U}}\right)^{\delta}\right)\right]^{m_{i, w}^{i n c t}}
\end{array}
$$

And the additional revenue generated by admitting LPUs for both the proposed Modified Dixit and Dixit models can be computed as

$$
\begin{gathered}
\boldsymbol{R}=\sum_{i=1}^{N_{L P U}} \alpha(t)_{i-\text { inct }}^{L P U} * P_{\text {util }}^{L P U} \\
p_{\text {inct }}^{L P U}=p_{l, \text { inct }}^{\text {nrtps }}=p_{q, \text { inct }}^{B E} \\
P_{\text {inct }}^{H P U}=p_{i, \text { inct }}^{U G S}=p_{i, \text { inct }}^{r t p s}=p_{k, \text { inct }}^{\text {ertps }} \\
\alpha(t)_{i-\text { inct }}^{L P U}=\alpha(t)_{l, \text { inct }}^{\text {nrtps }}=\alpha(t)_{q, \text { inct }}^{B E}
\end{gathered}
$$

Where,

$p_{i, \text { inct }}^{U G S}, p_{i, \text { inct }}^{r t p s}, p_{k, \text { inct }}^{\text {ertps }}$, are Reference Incentive flat prices payable by admitted UGS, rtps, ertps traffic; $P_{\text {inct }}^{H P U}=$ Reference Incentive flat Price payable by admitted High Priority Users; $p_{l, \text { inct }}^{n r t p s}, p_{q \text {,inct }}^{B E}$ Incentive Price payable by admitted nrtps, and BE traffic; $p_{\text {inct }}^{L P U}$ Incentive Price payable by admitted Low Priority Users; $\alpha(t)_{l, \text { inct }}^{n r t p s}, \alpha(t)_{q, \text { inct }}^{B E}$, Instantaneous Bandwidth Utilization by nrtPs and BE traffic in an incentive region; $\alpha_{i, w}^{H P U}$ is the Total Bandwidth Utilization by High Priority Users; $\alpha_{i, w}^{L P U}$ is the Total Bandwidth Utilization by Low Priority Users; $\alpha_{i, w}^{i c}$ is the Incentive Cut-off Bandwidth Utilization $\alpha_{i, w}^{t h}$ is the Threshold for Bandwidth Utilization; $m_{i, w}^{i n c t}$ is the Price Leveling Factor 
(PLF) in the incentive region and this provides additional flexibility to the Network Service Providers (NSPs) to modify their LPU pricing curves in the incentive region; $N_{l, i n c t}^{L P U}=$ $N_{l, \text { inct }}^{\text {nrtps }}+N_{\text {q,inct }}^{B E}$ is the total Number of admitted nrtPs and BE traffic in the incentive region.

Figures 2 and 3 depict the whole concepts of the Dixit and proposed modified Dixit. These two pricing models inherently handle LPU admission control by dynamically modulating the prices with respect to the fixed reference HPU price and based on the HPU demand. The LPU price normalized is plotted against the bandwidth utilization (BU) with and without HPU utilization having a $\mathrm{PLF}=0,0.1,1$, and 5 .

HPU price is the reference price used in these models to reflect the monetary incentives or penalties charged to the LPUs. The charging mechanism is assumed to use "per minute pricing (in minutes)" for real time applications and "per fixed data block pricing (unit: MB)" for this reference PU price, and this can be best represented by the "Prepaid/Pay-as-you-go" type services available in current wireless markets [4]. The $\mathrm{BU}$ from 0 to 0.7 is called the incentive region, while, the interval 0.7 to 0.9 is called the penalty region. The $\mathrm{BU}$ curves for $\mathrm{PLF}=0$ is a straight line at LPU $=0.5$. This indicates the HPU reference price above which the BU price is increased exponentially to discourage the utilization above incentive cut off limit. Other values of Incentive cut-off and threshold could be chosen by the NSPs for revenue maximization in a competitive environment.

\section{THE BEHAVIOR OF THE DIXIT MODEL}

Figure 2 shows the LPU price normalized versus the bandwidth utilization (BU) for Dixit without HPU utilization having a $P L F=0,0.1,1$, and 5 . In the Dixit model, the price increment is the multiples of the HPU reference price and it goes to infinity while approaching the BU threshold limit of 0.9. In the incentive region the lower PLF corresponding to the values of 0 and 0.1 generates BU curves with higher revenue than those with PLF $=1$ and 5. While in the congestion region, the reverse is the case. This is the scenario when there is no any HPU utilization. The short coming of this model is that we cannot do congestion control by tuning the PLFs in the congestion region as the price overshoots to infinity as BU approaches the threshold limit of 0.9 .

\section{THE BEHAVIOR OF THE PROPOSED MODIFIED DIXIT}

Figure 3 shows the LPU price normalized versus the bandwidth utilization (BU) for the proposed modified Dixit with HPU utilization having a PLF $=0,0.1,1$, and 5. The BU curves for $P L F=0$ is a straight line at $L P U=0.5$, this indicates, the HPU reference price above which the BU price is increased exponentially to discourage the utilization above incentive cut off limit. In the proposed modified Dixit model, the price increment is also the multiples of the HPU reference price and it goes to infinity while approaching the $\mathrm{BU}$ threshold limit of 0.9. In the incentive region the lower PLF corresponding to the values of 0 and 0.1 generates BU curves with higher revenue than those with PLF $=1$ and 5. While in the congestion region, the reverse is the case. The difference in this scenario is that there is an initial HPU bandwith utilization of 0.4. The short coming of this model is like the Dixit model where we cannot do congestion control by tuning the PLFs in the congestion region as the price overshoots to infinity as BU approaches the threshold limit of 0.9 . The advantage of this model over the conventional Dixit model is that there is fairness as the user would only be charged when he starts to utilize the bandwidth.

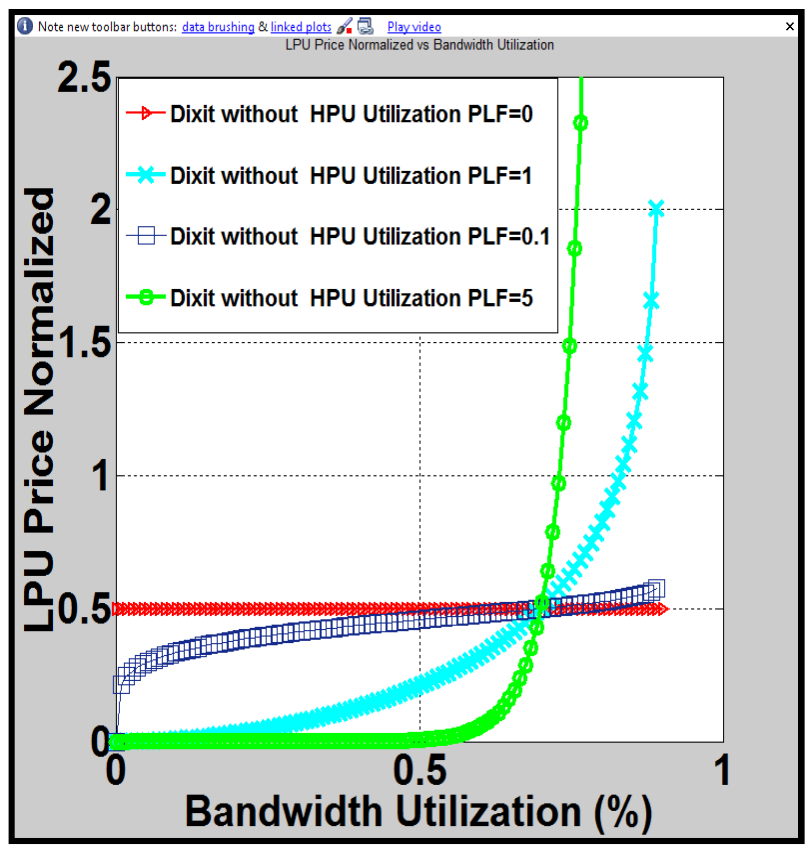

Figure 2: LPU BW Price Normalized vs. Bandwidth Utilization

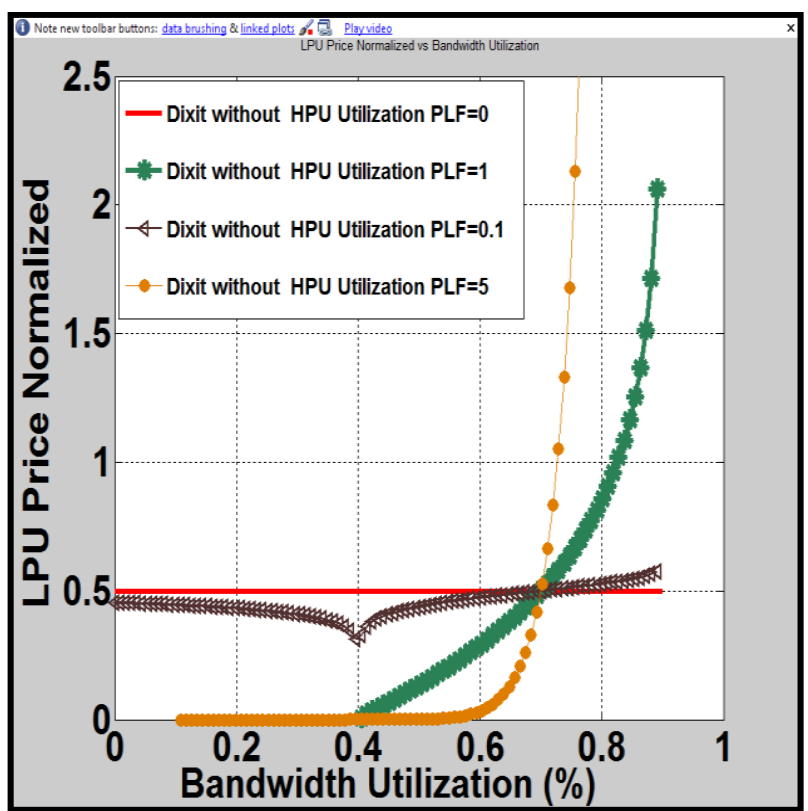

Figure 3: LPU BW Price Normalized vs. Bandwidth Utilization

\section{RESOURCE EFFICIENCY INDEX}

To determine jointly the optimal CAC strategy at the best pricing scheme, we introduce the network provider's point of view, mainly in terms of resource efficiency index which is defined as the ratio of the potential revenue and the total resource allocated. Revenue is not only a function of BW price, but depends also on the users' degree of satisfaction, i.e. perceived QoS as depicted in microeconomics by utility functions, and their willingness to pay. This is called the 
acceptance probability. The pricing strategies we shall focus on are flat and service-based pricing. In the former, a user pays a fixed amount of money per time unit, regardless of the changing performance he might experience throughout the duration of his call.

We use the metric in [6] for the comparative and performance analysis of the proposed model and the existing model

$$
E=\frac{R}{B}
$$

$\mathrm{E}=$ Resource Efficiency Index,$R=$ Total revenue generated from the admitted users and $\mathrm{B}=$ Total Allocated Resources to the admitted users

\section{PERFORMANCE ANALYSIS}

In this section, the performance of the proposed new BW price based bandwidth management is evaluated by comparing it with the model in [4].

Matlab simulations were carried out in order to analyze the proposed pricing mechanisms. Revenue, bandwidth utilization and resource efficiency index are the parameters chosen for the analysis. Table 1 depicts the whole parameterization of the Dixit and the proposed modified Dixit. The NSPs need to have a mechanism for price flexibility to enable price adjustment for profit maximization and for competing with the prices set by the other NSPs in the region. To aid this purpose, the Price Leveling Factor (PLF) is introduced [4]. This factor allows the adjustment in LPU prices according to NSP requirements, keeping the configuration parameters ai,ic and $\alpha i$,th set by the NSP unchanged. The PLFs for these simulations are $0,0.1,1$, and 5 . These two pricing models inherently handle LPU admission control by dynamically modulating the prices with respect to the fixed reference HPU price and based on the HPU demand. HPU price is the reference price used in these models to reflect the monetary incentives or penalties charged to the LPUs. The BU from 0.4 to 0.7 is called the incentive or non congestion region, while, the interval 0.7 to 0.9 is called the penalty region. The BU curves for $\mathrm{PLF}=0$ is a straight line at $\mathrm{LPU}=0.5$, this indicates, the HPU reference price above which the BU price is increased exponentially to discourage the utilization above the incentive cut off limit. Other values of Incentive cut-off and threshold could be chosen by the NSPs for revenue maximization in a competitive environment.

Table 1: The Simulation Parameters for the Proposed Modified Dixit and Dixit S., Et al. Model

\begin{tabular}{|l|l|}
\hline Parameters & Settings \\
\hline & \\
Bandwidth Utilization Threshold & 0.9 \\
Beserved Bandwidth Utilization & 0.1 \\
HPU Bandwidth Utilization & 0.7 \\
HPU reference normalized Price & 0.5 \\
Price leveling Factors & $0,0.1,0.5$, and 1 \\
HPU maximum Price & Infinity \\
\hline
\end{tabular}

Figure 4 shows cumulative revenue (CR) of the BS versus the cumulative bandwidth utilization (CBU) for both Dixit without HPU utilization and the proposed modified dixit with
HPU utilization both having a PLF=5. The CR for both the two models is below $\$ 50$ for a CBU ranging from $0 \%$ to $40 \%$. The \% improvement of the CR generated by the proposed modified dixit model with HPU utilization over CR generated by the Dixit model without HPU utilization at PLF=5 is $100 \%$. This could be as a result of much higher CBU in the proposed modified dixit

Figure 5 shows the cumulative revenue (CR) of the $\mathrm{BS}$ versus the cumulative bandwidth utilization (CBU) for both Dixit without HPU utilization and the proposed modified dixit with HPU utilization both having a $P L F=0$. The $C R$ for both the two models increases from 0 to $\$ 23$ for a CBU ranging from $0 \%$ to $46 \%$. For the proposed model there seems to be an additional $\mathrm{CR}$ of $\$ 4$ for an additional CBU of $9 \%$.

Figure 6 shows the cumulative revenue (CR) of the BS versus the cumulative bandwidth utilization (CBU) for both Dixit without HPU utilization and the proposed modified dixit with HPU utilization both having a PLF=0.1. The CR for both the two models increases from 0 to $\$ 23$ for a CBU ranging from $0 \%$ to $46 \%$. For the proposed modified Dixit model there seems to be an additional CR of $\$ 4$ for an additional CBU of $9 \%$.

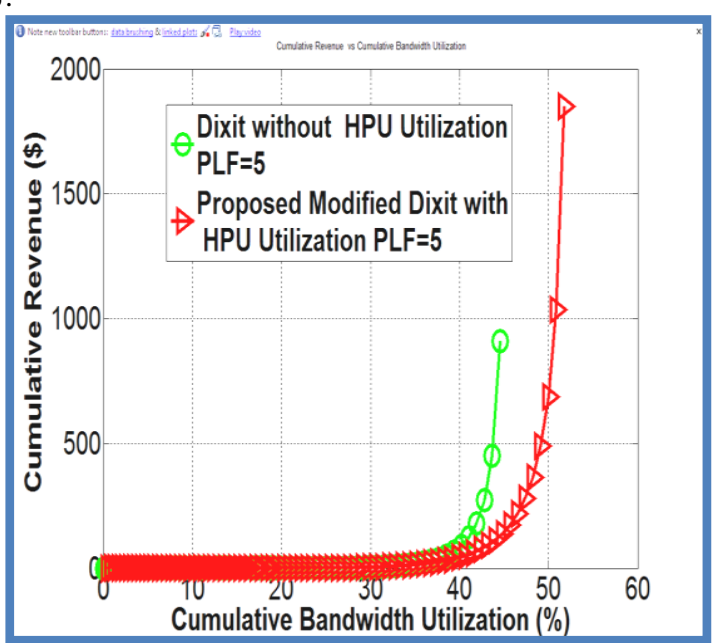

Figure 4: Cumulative Revenue vs. Cumulative Bandwidth Utilization

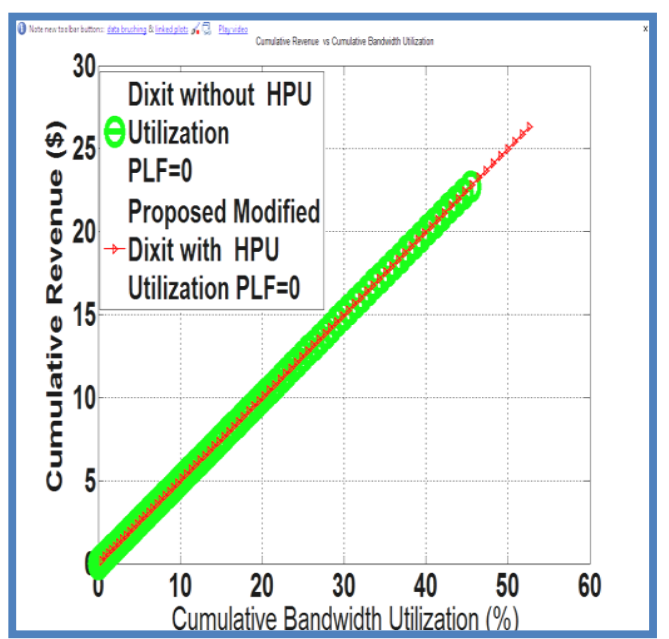

Figure 5: Cumulative Revenue vs. Cumulative Bandwidth Utilization 


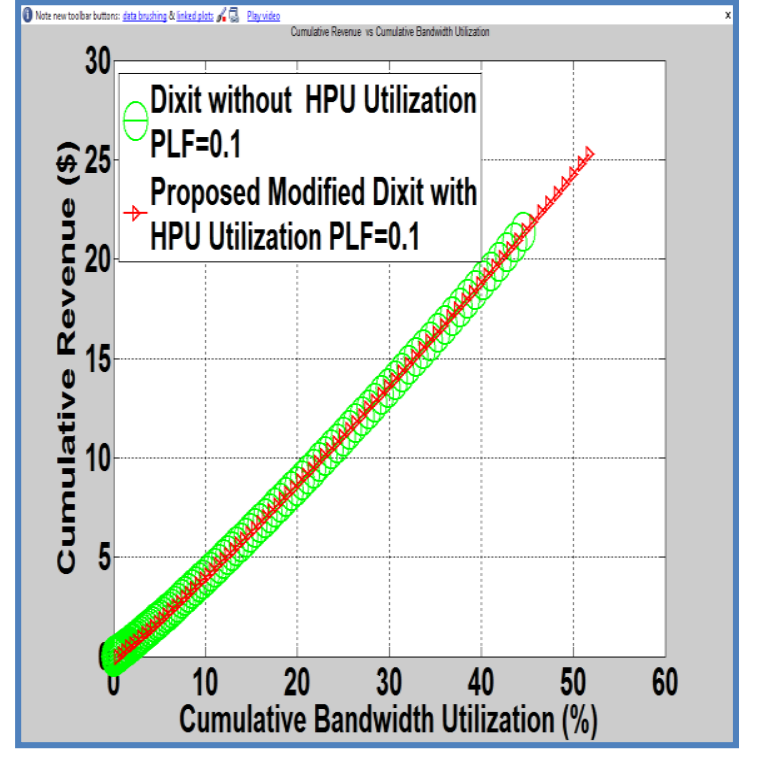

Figure 6: Cumulative Revenue vs. Bandwidth Utilization

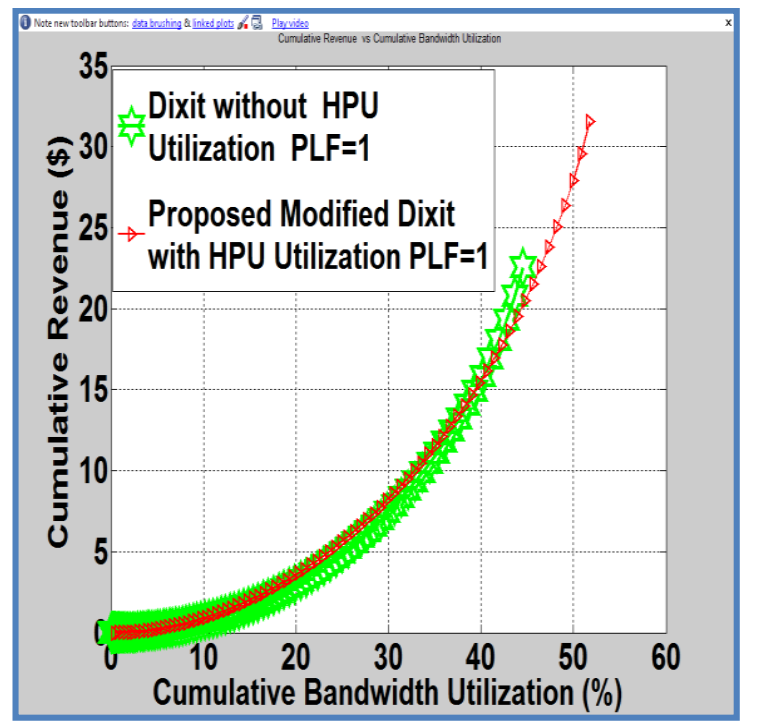

Figure 7: Cumulative Revenue vs. Bandwidth Utilization

Figure 7 shows the cumulative revenue (CR) of the BS versus the cumulative bandwidth utilization (CBU) for both Dixit without HPU utilization and the proposed modified dixit with HPU utilization both having a PLF=1. The CR for both the two models increases exponentially from 0 to $\$ 23$ for a CBU ranging from $0 \%$ to $46 \%$. For the proposed model there seems to be an additional CR of $\$ 10$ for an additional CBU of $7 \%$.

Figure 8 shows the Cumulative Resource efficiency index (CRI) of the BS versus the cumulative revenue (CR) for the Dixit without HPU utilization, and the proposed modified Dixit with HPU utilization both having a PLF $=0$. The CRI of Dixit without HPU utilization and the proposed modified Dixit with HPU utilization is also 0.5 across the range of CR from $\$ 0$ to $\$ 23$ and $\$ 0$ to $\$ 53$, respectively.

Figure 9 shows the Cumulative Resource efficiency index (CRI) of the BS versus the cumulative revenue (CR) for the Dixit without HPU utilization, and the proposed modified Dixit with HPU utilization both having a PLF $=1$. The CRI of Dixit without HPU utilization and the proposed modified
Dixit with HPU utilization increases exponentially from 0 to 0.62 across the range of CR from $\$ 0$ to $\$ 23$ and $\$ 0$ to $\$ 32$, respectively. The \% improvement of the CRI generated by the proposed modified dixit model with HPU utilization over that of Dixit model without HPU utilization at PLF $=1$ is $24 \%$.

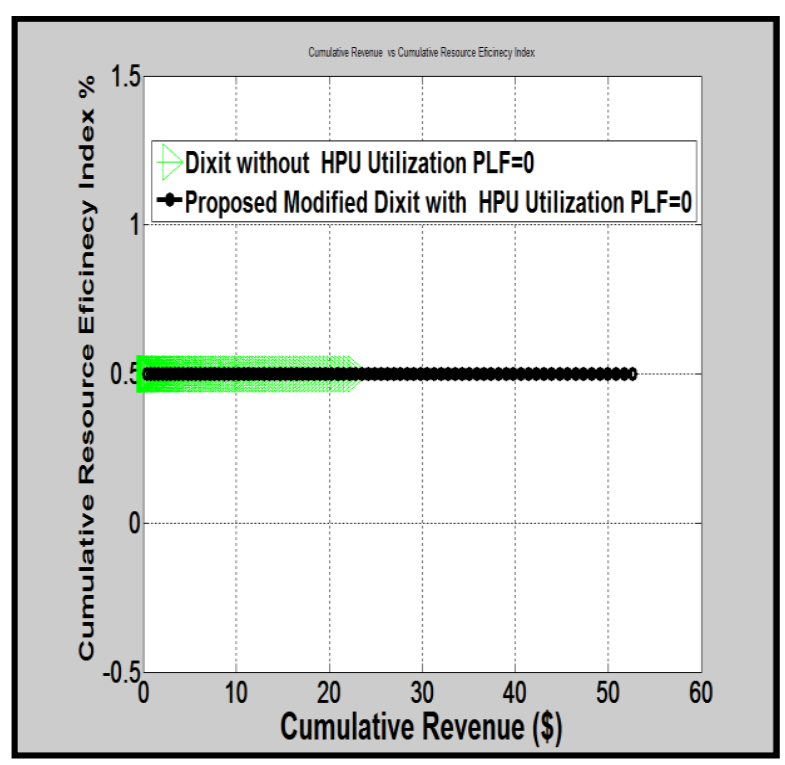

Figure 8: Cumulative Resource Efficiency Index vs. Cumulative Bandwidth Utilization

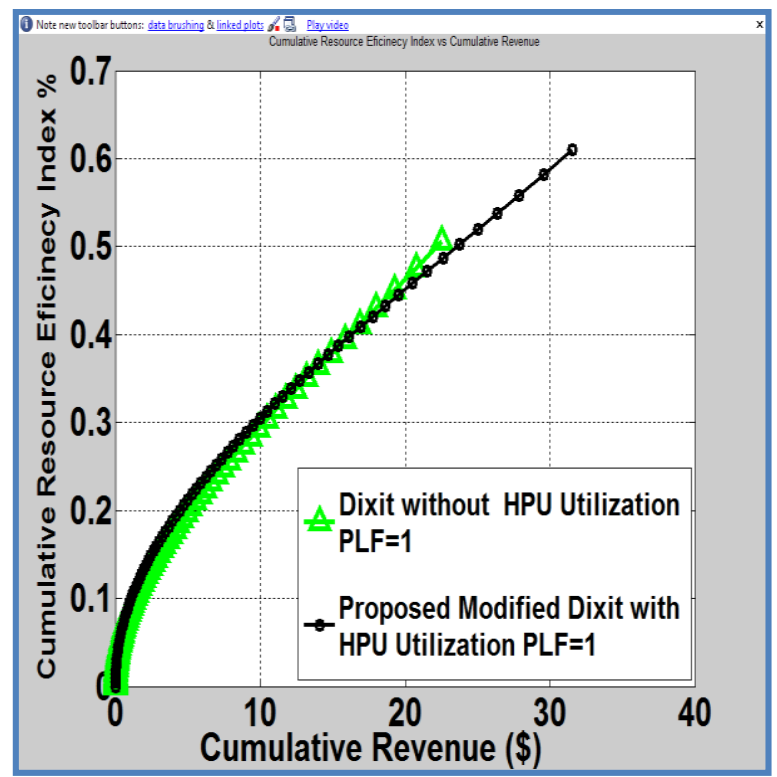

Figure 9: Cumulative Resource Efficiency Index vs. Cumulative Revenue

Figure 10 shows the Cumulative Resource efficiency index (CRI) of the BS versus the cumulative revenue (CR) for the Dixit without HPU utilization and proposed modified Dixit with HPU utilization both having a PLF $=0.1$. The CRI of Dixit without HPU utilization increases exponentially from 0.2 to 0.48 across the range of CR from $\$ 0$ to $\$ 23$. The CRI of the proposed modified Dixit with HPU utilization increases exponentially from 0.2 to 0.5 across the range of CR from $\$ 0$ to $\$ 26$. At a CR of $\$ 23$, the \% improvement of the CRI generated by the proposed model with HPU utilization over that of Dixit model without HPU utilization at PLF $=0.1$ is $6.3 \%$. 
Figure 11 shows the Cumulative Resource efficiency index (CRI) of the BS versus the cumulative revenue (CR) for the Dixit without HPU utilization and proposed modified Dixit with HPU utilization both having a PLF=5. The CRI of Dixit without HPU utilization increases linearly from 0 to 20 across the range of CR from $\$ 0$ to $\$ 900$. The CRI of the proposed modified Dixit with HPU utilization also increases linearly from 0 to 35 across the range of CR from $\$ 0$ to $\$ 1850$. At a range of CR 0 to $\$ 900$, the Dixit without HPU utilization shows some significant values that are above the proposed model. The \% improvement of the CRI generated by the proposed modified Dixit with HPU utilization over that of Dixit model without HPU utilization at PLF $=5$ is $71 \%$.

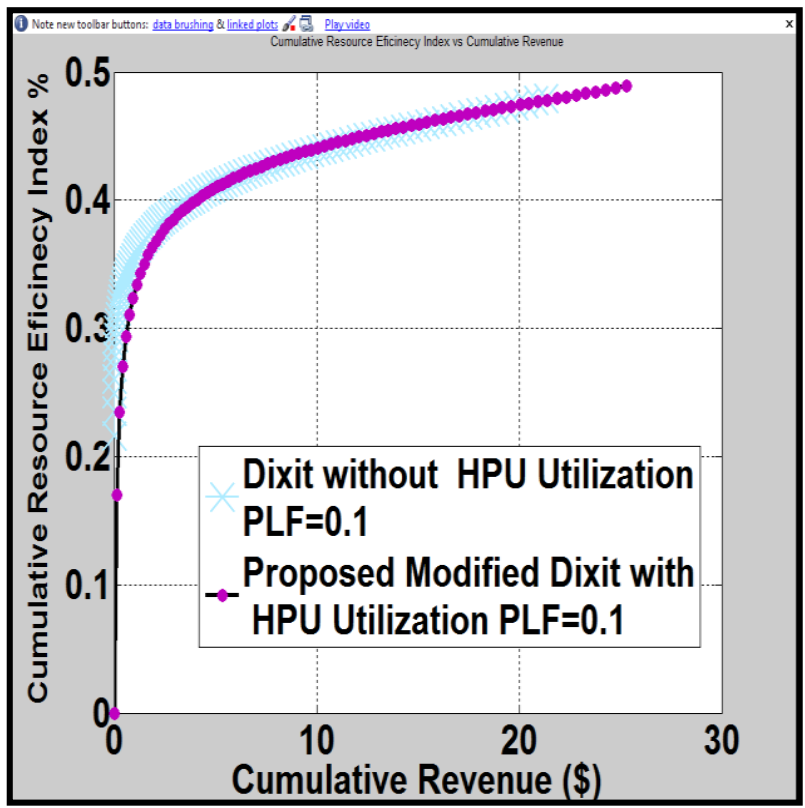

Figure 10: Cumulative Resource Efficiency Index vs. Cumulative Revenue

\section{CONCLUSIONS}

In this paper, a mathematical model for an efficient dynamic pricing is developed for optimal network resource utilization using a modified log barrier function in Mobile WiMAX network. The percentage improvement of the Cumulative Resource Efficiency Index (CREI) generated by the proposed model over the existing model ranges between $6 \%$ and $71 \%$ depending on the values of the Price Leveling Factor (PLF). The scheme proved to generate more revenue per Bandwidth Utilization. For further improvement, a model with HPU utilization and a price increment which approaches 1 above the HPU reference price instead of infinity could be developed. Congestion control could be possible by tuning the values of PLFs according to the introduced concept of the proposed utility and acceptance probability models.

\section{ACKNOWLEGEMENT}

We wish to thank the management of Nigerian Communications Commission NCC and Electrical Department of Bayero University Kano for their financial support. We also extend our gratitude to Dr. S. S. Adamu, Dr. Muhammad Ajiya, Dr. Sabo Ibrahim Birnin Kudu and Dr. Sulaiman Garba for their intellectual guidance and support.

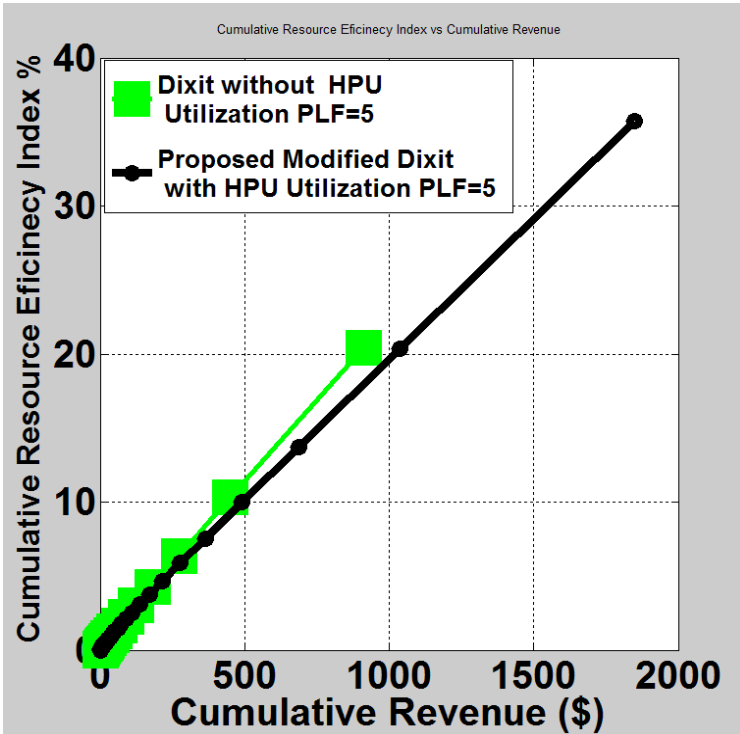

Figure 11: Cumulative Resource Efficiency Index vs. Cumulative Revenue

\section{REFERENCES}

[1] Shuaibu, D.S. (2012). Radio Resource Management for Mobile WiMAX Network. A Thesis Submitted in partial fulfillment of the requirements of Universiti Technologi Malaysia for the Degree of Doctor of Philosophy.

[2] Ramad, K. and Jain, R. (2008). WiMAX System evaluation Methodology. WiMAX Forum, version 2.1 July 2008.

[3] Akyildiz I., Lee W., Vuran M. and Mohanty S. (2006). NeXt generation/dynamic spectrumaccess/ cognitive radio wireless networks. A survey, Computer Networks, Vol. 50, no. 13, pp. 2127-2159.

[4] Mowafi, M. Y., Al-Mistarihi, M. F. and Marei, M. S (2012). A Dual Usage of Cognitive Radio in Managing the WiMAX Band width MIPRO /CTI.

[5] Dixit, S., Periyalwar S. and Yanikomeroglu, H. (2013). Secondary User Access in LTE Architecture Based on a Base-Station-Centric Framework With Dynamic Pricing, IEEE Transactions on Vehicular Technology, Vol. 62, no. 1 , pp. 284-296.

[6] Badia L., Lindstrom J. and Zorzi M (2004). On Utilitybased Radio Resource Management with and without Service Guarantees, Proceedings of the 7th ACM International Symposium on Modeling, Analysis and Simulation of Wireless and Mobile Systems Venice, Italy, pp. 244-251.

[7] Vuong Q.N (2008). Mobility Management in 4G Wireless Heterogeneous Networks. A Thesis Submitted in partial fulfillment of the requirements of Universite D'evry Val-D'essonne for the Degree of Doctor of Philosophy. 